\title{
Reduction of radiation exposure during transcatheter edge-to-edge mitral valve repair
}

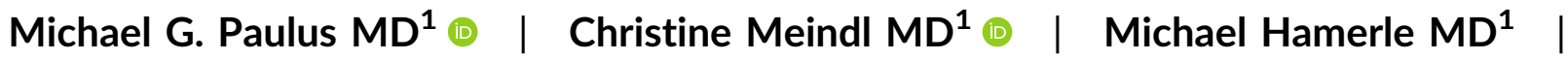 \\ Christian Schach MD ${ }^{1}$ | Lars S. Maier MD ${ }^{1}$ | Kurt Debl MD ${ }^{1}$ | \\ Christoph Birner MD ${ }^{2}$ | Bernhard Unsöld MD ${ }^{1}$
}

${ }^{1}$ Department of Internal Medicine II, University Hospital Regensburg, Regensburg, Germany

${ }^{2}$ Department of Internal Medicine I, Klinikum St. Marien, Amberg, Germany

\section{Correspondence}

Michael G. Paulus, Department of Internal Medicine II, University Hospital Regensburg, Franz-Josef-Strauß-Allee 11, 93053

Regensburg, Germany.

Email:michael.paulus@ukr.de

\begin{abstract}
Background: Transcatheter mitral valve repair is an increasingly used therapy for mitral regurgitation which requires fluoroscopic guidance. Limiting radiation exposure during lengthy procedures is important for both patient and operator safety. This study aimed to investigate radiation dose during contemporary use of MitraClip implantation and the effects of a dose reduction program.

Methods: A total of 115 patients who underwent MitraClip implantation were prospectively enrolled in a single-center observational study. During the inclusion period, our institution adopted a radiation dose reduction program, comprising lowering of fluoroscopy pulse rate and image target dose. The first 58 patients were treated with conventional fluoroscopy settings, while the following 57 patients underwent the procedure with the newly implemented low dose protocol.

Results: Radiation dose area product significantly decreased after introduction of the low dose protocol (693 [366-1231] vs. 2265 [1517-3914] cGy·cm², $p<0.001$ ). After correcting for fluoroscopy time, gender and body mass index, the low dose protocol emerged as a strong negative predictor of radiation dose $(p<0.001)$, reducing dose area product by $64 \%$ (95\% confidence interval [57-70]). Device time, device success, and procedural safety did not differ between the normal dose and low dose group. Furthermore, the low dose protocol was not associated with an increased incidence of a combined endpoint consisting of death, repeat intervention, or heart surgery during 12 months follow-up.

Conclusion: Reduction of radiation exposure during transcatheter mitral valve repair by $64 \%$ is feasible without affecting procedural success or safety.
\end{abstract}

\section{KEYWORDS}

fluoroscopy, mitral regurgitation, radiation dosage 


\section{1 | INTRODUCTION}

Medical procedures are the most important source of artificial ionizing radiation, which is known to constitute a non-negligible hazard for public health. Cardiology itself accounts for $40 \%$ of the total medical radiation burden excluding radiation oncology. ${ }^{1}$ Among the imaging methods in cardiovascular medicine, X-ray fluoroscopy has both the largest variation in dose per procedure and the highest potential for extensive radiation exposure. Likewise, fluoroscopic guided procedures are responsible for half of the total radiation dose in cardiology patients, while only accounting for $12 \%$ of all performed radiological examinations. ${ }^{2}$ Therefore, monitoring radiation exposure and optimizing the parameters which affect radiation dose is essential when applying this imaging modality in routine clinical care. ${ }^{3}$ Apart from coronary angiography, several newly emerged methods of percutaneous treatment of structural heart disease make use of fluoroscopic guidance. Among these, transcatheter edge-to-edge mitral valve repair is an established therapy for mitral regurgitation in high-risk patients with proven safety and efficacy. ${ }^{4,5}$ Although fluoroscopy time can exceed one hour in lengthy and complex procedures, ${ }^{6}$ little is known about the radiation burden associated with transcatheter mitral valve repair. In the perspective of the increasing usage of minimal-invasive mitral valve therapy, this study aimed to investigate radiation exposure during MitraClip implantation and the effects of an institutional dose reduction program.

\section{2 | METHODS}

\section{1 | Study design}

From August 2017 to April 2020, we prospectively enrolled patients in a single-center, investigator-driven observational study at the University Heart Center Regensburg. Qualifying inclusion criterion was moderate-to-severe or severe mitral regurgitation treated with transcatheter edge-to-edge mitral valve repair using the MitraClip system. Cases with failure to implant a device, additional non-mitral intervention in the same session or missing data on radiation dose were excluded from analysis. The decision for transcatheter mitral valve repair in each patient was made by an interdisciplinary Heart Team consisting of an interventional cardiologist, a cardiac surgeon, and an anesthesiologist. The procedures were performed by two experienced primary operators (C.B. and B.U.) and secondary operators from the team (C.B., B.U., K.D., C.M., and C.S.) using the MitraClip NT, NTR, or XTR system (Abbott Vascular) at their discretion. Mitral valve repair was carried out as described elsewhere. ${ }^{7}$ Transesophageal echocardiographic guidance was provided by two operators (C.M. and C.S.) using 2D- and 3D-imaging on Philips Epiq CVx or iE-33 ultrasound systems (Philips Medical Systems) equipped with matrix array transducers. During the study period, echocardiographic modalities remained constant. The study did not receive external funding and was approved by the local ethics committee. All participants gave written consent before study enrollment.
All participants underwent comprehensive clinical and echocardiographic assessment at baseline. Residual mitral regurgitation was quantified before discharge by transthoracic echocardiography. Mitral regurgitation grading was based on color and continuous wave Doppler examination in accordance with current guidelines. ${ }^{8,9}$ Regurgitation grade was scored from 1 to 4 (1: mild, 2: mild-tomoderate, 3: moderate-to-severe, and 4: severe). Device time was defined as the time from guide catheter insertion to guide catheter removal. Patients were followed up for up to twelve months by outpatient visits, phone calls, and contact with the primary care physician. The incidence of death, repeat mitral valve intervention, or heart surgery was defined as the combined endpoint for assessing midterm clinical outcome.

\section{2 | Fluoroscopy modalities and dose reduction protocol}

All procedures were performed with the same X-ray equipment consisting of a flat panel angiography system (AXIOM Artis, Siemens Healthineers). Fluoroscopic guidance was mainly used for transseptal puncture, advancing the guide catheter into the left atrium, and steering of the MitraClip towards the mitral valve. During grasping of the valve leaflets, fluoroscopy was used to maintain correct rotation of the device. Establishment of final arm angle, deployment of the clip, and removal of the guide catheter from the left atrium were also visualized by fluoroscopy. Total radiation dose during the procedure was recorded by a dose area product meter integrated in the X-ray tube output which was calibrated for accuracy during annual inspections.

From the start of patient enrollment in August 2017-July 2018, the manufacturer's default X-ray settings for fluoroscopyguided procedures in cardiology were applied: pulsed fluoroscopy with a pulse rate of $6 / \mathrm{s}$, automatic exposure control with a target dose of $45 \mathrm{nGy}$ per image, normal dose x-ray generator power curve (70 kV $10 \mathrm{R} \mathrm{AF} 3 \mathrm{~kW}$ ); cine-acquisition with a frame rate of $15 / \mathrm{s}$, automatic exposure control with a target dose of $170 \mathrm{nGy}$ per image. Starting in August 2018, our institution implemented a radiation dose reduction program for transcatheter mitral valve repair, establishing a 'low dose' fluoroscopy protocol. Based on previous studies on radiation dose reduction in interventional cardiology, ${ }^{10,11}$ we decreased image frame rate and target dose as follows: (i) reduction of the pulse rate of fluoroscopy to $3 / \mathrm{s}$, (ii) reduction of the pulse rate of cine-acquisition to $7.5 / \mathrm{s}$, and (iii) reduction of the target dose of pulsed fluoroscopy to $36 \mathrm{nGy}$ per image. Additionally, we selected an X-ray generator power curve recommended by the manufacturer for low dose imaging in electrophysiological or pediatric examinations $(100 \mathrm{kV} 10 \mathrm{R}$ AF $2 \mathrm{~kW}$ small focus). A comparison of pulsed fluoroscopy image quality with low dose and normal dose settings is shown in Figure 1 and Moving image S1 and S2. Operators were encouraged to adhere to the settings but were allowed to make adjustments during the procedure if clinically deemed necessary. 


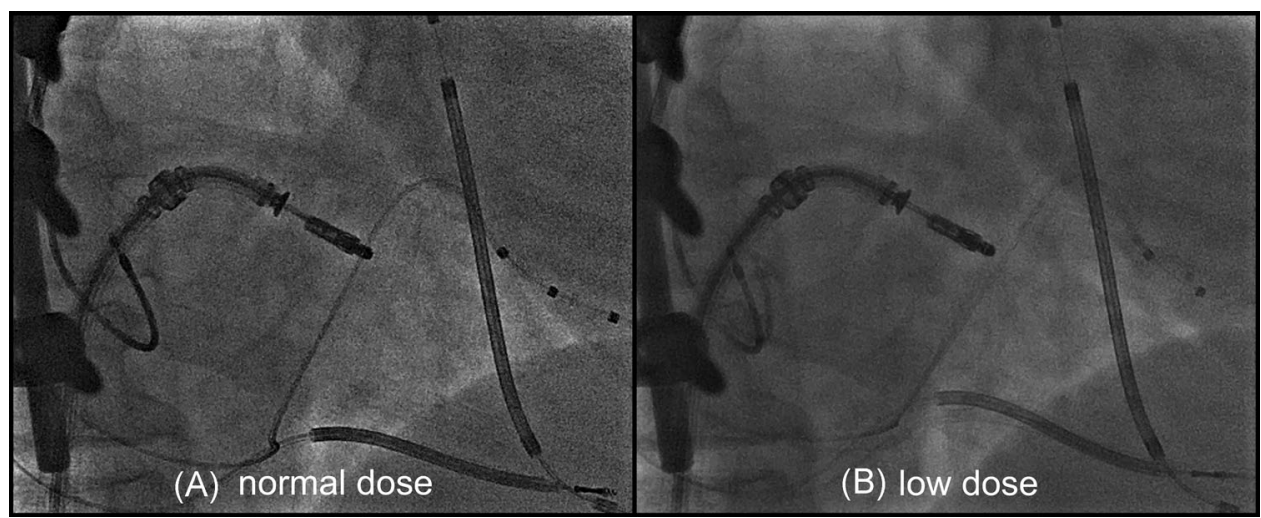

FIGURE 1 Comparison of pulsed fluoroscopy image quality during transcatheter mitral valve repair with (A) normal dose and (B) low dose settings. In addition to the MitraClip device, the image features multiple pacemaker/defibrillator leads and osteosynthetic material not related to the procedure

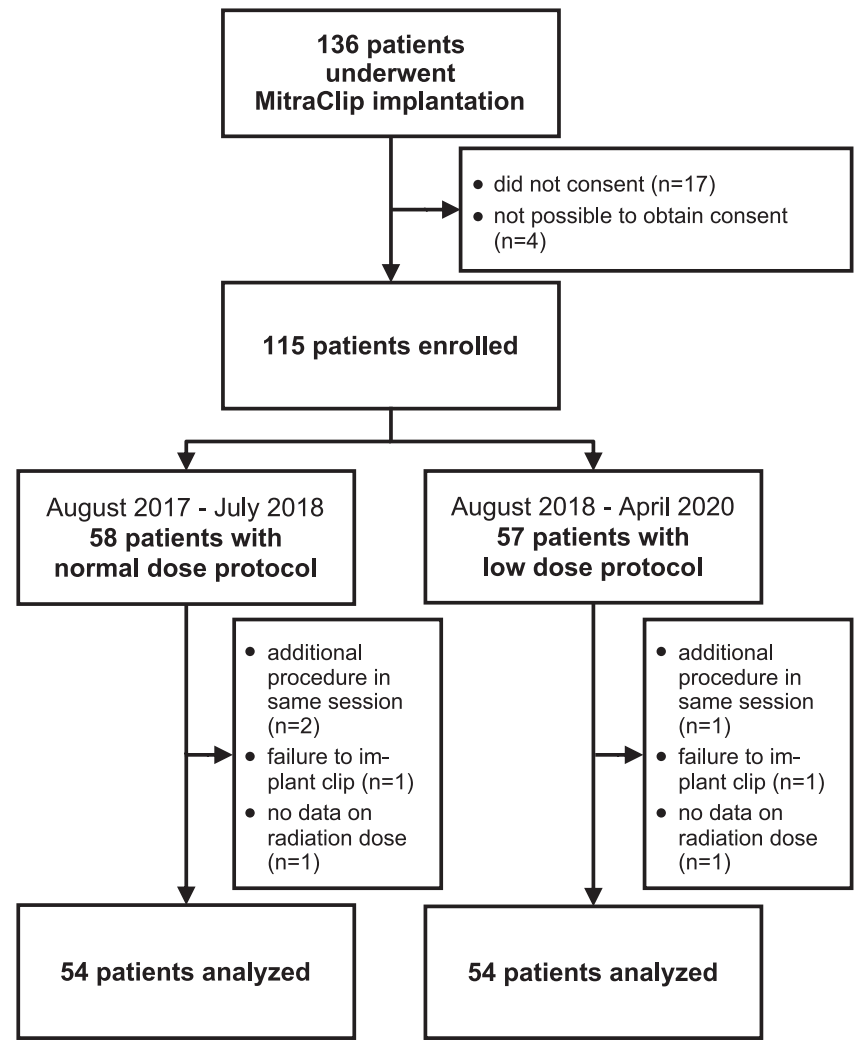

FIGURE 2 Flow diagram depicting the study inclusion process

\section{3 | Statistical analysis}

Continuous variables are reported as mean \pm standard deviation when normally distributed, continuous variables with skewed distribution as median with interquartile range (first quartile-third quartile). Categorical variables are presented in numbers and percentages. Differences between the low dose and normal dose group were assessed using students t-test for continuous data, Mann-Whitney-U test for ordinal data and Pearson's $\chi^{2}$ test for nominal data. Differences in binary variables were analyzed applying Fisher's exact test. The incidence of the combined endpoint death, repeat intervention, or heart surgery in the normal dose and low dose group was compared by a log-rank test. Multiple linear regression was used to identify independent predictors of radiation exposure. Due to the highly skewed distribution, dose area product was transformed into its natural logarithm. Selection of predictors was based on clinical considerations and included mitral regurgitation grade at baseline, number of implanted devices, body mass index, gender, radiation protocol, and fluoroscopy time. To preserve linear correlation with the dose area product, fluoroscopy time was entered into the model as its natural logarithm. Percent changes of binary predictor variables were calculated as the natural exponential function of the regression coefficient. Normal distribution and homoscedasticity of residuals were verified with residual plots. Durbin-Watson statistic was used to check for autocorrelation. Predictors were tested for collinearity by calculating variance inflation factors. Outlier analysis was performed by reviewing deleted studentized residuals and Cook's distances. A two-sided $p$-value $<0.05$ was considered statistically significant. All statistical analyses were performed using SPSS Statistics 25 (IBM).

\section{3 | RESULTS}

\section{1 | Study population}

Of 136 performed MitraClip procedures between August 2017 and April 2020, a total of 115 patients were enrolled in the study (Figure 2). The first 58 participants underwent MitraClip implantation with the normal fluoroscopy protocol, whereas the following 57 patients were treated with the newly implemented low dose protocol. Failure to implant the device due to anatomical reasons occurred once in the normal dose group and once in the low dose group and was not associated with a complication. All procedures in the low dose group were carried out without intraprocedural deviation from 
TABLE 1 Baseline characteristics of the study population

\begin{tabular}{|c|c|c|c|}
\hline \multirow[b]{2}{*}{ Variable } & \multicolumn{2}{|c|}{ Fluoroscopy protocol } & \multirow[b]{2}{*}{$p$-value } \\
\hline & $\begin{array}{l}\text { Low } \\
\text { dose }(n=54)\end{array}$ & $\begin{array}{l}\text { Normal } \\
\text { dose }(n=54)\end{array}$ & \\
\hline Age, years & $76 \pm 8$ & $76 \pm 7$ & 0.88 \\
\hline Female gender & $16(30 \%)$ & $26(48 \%)$ & 0.08 \\
\hline Body mass index, $\mathrm{kg} / \mathrm{m}^{2}$ & $26.3 \pm 5.8$ & $26.2 \pm 3.6$ & 0.96 \\
\hline Heart failure entity & & & 0.07 \\
\hline HFpEF & $24(44 \%)$ & 35 (65\%) & \\
\hline HFmrEF & 12 (22\%) & $5(9 \%)$ & \\
\hline HFrEF & 18 (33\%) & $14(26 \%)$ & \\
\hline Coronary artery disease & 37 (69\%) & 25 (46\%) & 0.03 \\
\hline $\begin{array}{l}\text { Dilated } \\
\text { cardiomyopathy }\end{array}$ & $5(9 \%)$ & $9(17 \%)$ & 0.39 \\
\hline Atrial fibrillation & 40 (74\%) & $31(57 \%)$ & 0.10 \\
\hline Diabetes mellitus & $16(30 \%)$ & $15(28 \%)$ & 1.00 \\
\hline EuroSCORE II,\% & $6.0[3.2-10.9]$ & $5.2[3.4-7.6]$ & 0.65 \\
\hline GFR, $\mathrm{ml} / \mathrm{min}$ & $43 \pm 20$ & $47 \pm 22$ & 0.31 \\
\hline NTproBNP, pg/ml & $\begin{array}{l}2780 \\
{[1561-8045]}\end{array}$ & $\begin{array}{l}2732 \\
{[847-4638]}\end{array}$ & 0.21 \\
\hline NYHA functional class & & & 0.37 \\
\hline I & 0 & 0 & \\
\hline II & $6(11 \%)$ & $4(8 \%)$ & \\
\hline III & 40 (74\%) & 47 (89\%) & \\
\hline IV & 8 (15\%) & 2 (4\%) & \\
\hline MR Grade & & & 1.00 \\
\hline 1 & 0 & 0 & \\
\hline 2 & 0 & 0 & \\
\hline 3 & $11(20 \%)$ & $11(20 \%)$ & \\
\hline 4 & 43 (80\%) & $43(80 \%)$ & \\
\hline $\begin{array}{l}\text { MR of secondary or } \\
\text { mixed etiology }\end{array}$ & 43 (80\%) & 35 (65\%) & 0.13 \\
\hline PISA EROA & $0.38 \pm 0.22$ & $0.44 \pm 0.24$ & 0.23 \\
\hline LVEF, \% & $43 \pm 15$ & $48 \pm 15$ & 0.14 \\
\hline LVEDV, ml & $177 \pm 73$ & $168 \pm 65$ & 0.50 \\
\hline $\begin{array}{l}\text { Left atrial volume index, } \\
\qquad \mathrm{ml} / \mathrm{m}^{2}\end{array}$ & $82 \pm 32$ & $82 \pm 37$ & 1.00 \\
\hline
\end{tabular}

Note: Continuous variables with normal distribution are expressed as mean $\pm S D$, continuous variables with skewed distribution as median [IQR]. Categorical variables are expressed as $n(\%)$.

Abbreviations: EROA, effective regurgitation orifice area; GFR, glomerular filtration rate; HFmrEF, heart failure with mid-range ejection fraction; $\mathrm{HFpEF}$, heart failure with preserved ejection fraction; HFrEF, heart failure with reduced ejection fraction; IQR, interquartile range; LVEDV, left ventricular end diastolic volume; LVEF, left ventricular ejection fraction; MR, mitral regurgitation; NYHA, New York Heart Association; PISA, proximal isovelocity surface area; $S D$, standard deviation. the low dose settings. Baseline characteristics are shown in Table 1. Mitral regurgitation severity and proportion of secondary etiology was similar in the low dose and normal dose group. Furthermore, both groups shared comparable age, perioperative risk, and functional status. Of note, body mass index was also similar in both groups.

\section{2 | Radiation exposure and procedural outcome}

After introduction of the low dose protocol, radiation dose area product dropped from 2265 [1517-3914] to 693 [366-1231] $\mathrm{cGy} \cdot \mathrm{cm}^{2}(p<0.001$, Table 2 and Figure 3$)$. Concurrently, fluoroscopy time decreased from $25[15-36]$ to 17 [13-23] $\mathrm{min}$ $(p=0.02)$. Dose area product per fluoroscopy time also strongly decreased from $110[80-140]$ to 38 [22-61] cGy $\cdot \mathrm{cm}^{2} / \mathrm{min}$ $(p<0.001)$, reflecting the adjustments in X-ray settings. At the same time, the low dose protocol did not prolong the procedure in terms of device time ( $p=0.77)$ while the number of implanted devices remained unchanged $(p=0.84)$. Residual mitral regurgitation before discharge was without significant difference between the low dose and normal dose group ( $p=0.75)$, demonstrating equivalent procedural success. Furthermore, usage of the low dose protocol was not associated with an increase in procedure-related complications.

\section{3 | Predictors of radiation exposure}

To identify predictors of radiation dose during transcatheter mitral valve repair, a multiple linear regression model was created (Table 3). Based on plausibility, mitral regurgitation grade at baseline, number of implanted devices, radiation protocol, fluoroscopy time, body mass index, and gender were included as predictor variables. Body mass index and fluoroscopy time independently predicted higher radiation exposure while female gender was associated with lower radiation dose. Mitral regurgitation grade at baseline or number of implanted devices did not influence radiation dose. The low dose protocol emerged as the strongest negative predictor of radiation dose, reducing dose area product by $64 \%$ (95\% confidence interval [57\%-70\%]).

\section{4 | Midterm clinical outcome}

To assess the effects of the low dose protocol on clinical outcome, patients were followed up for a median of 12 months. Results of the combined endpoint of death, repeat intervention, or heart surgery are shown in Figure 4. Cumulative freedom of event at twelve months was $87 \%$ in the normal dose and $94 \%$ in the low dose group, not revealing a significant difference $(p=0.54)$. Thus, introduction of the low dose protocol was not associated with worse midterm clinical outcome. 
TABLE 2 Procedural details of patients treated with MitraClip implantation

\begin{tabular}{|c|c|c|c|}
\hline \multirow[b]{2}{*}{ Variable } & \multicolumn{2}{|c|}{ Fluoroscopy protocol } & \multirow[b]{2}{*}{$p$-value } \\
\hline & Low dose & Normal dose & \\
\hline $\begin{array}{l}\text { Dose area product, } \\
\qquad \mathrm{cGy} \cdot \mathrm{cm}^{2}\end{array}$ & $\begin{array}{l}693 \\
\quad[366-1231]\end{array}$ & $\begin{array}{l}2265 \\
\quad[1517-3914]\end{array}$ & $<0.001$ \\
\hline Fluoroscopy time, min & 17 [13-23] & $25[15-36]$ & 0.02 \\
\hline $\begin{array}{l}\text { Dose area product per } \\
\text { fluoroscopy time, } \\
\mathrm{cGy} \cdot \mathrm{cm}^{2} / \mathrm{min}\end{array}$ & 38 [22-61] & $110[80-140]$ & $<0.001$ \\
\hline Device time, $\min$ & $45[30-70]$ & $55[35-80]$ & 0.77 \\
\hline No. of implanted devices & & & 0.84 \\
\hline 1 & 27 (50\%) & $30(56 \%)$ & \\
\hline 2 & $26(48 \%)$ & $23(43 \%)$ & \\
\hline 3 & $1(2 \%)$ & $1(2 \%)$ & \\
\hline $\begin{array}{l}\text { MR Grade post } \\
\text { intervention }\end{array}$ & & & 0.75 \\
\hline 1 & $38(70 \%)$ & $36(67 \%)$ & \\
\hline 2 & $12(22 \%)$ & $15(28 \%)$ & \\
\hline 3 & $4(7 \%)$ & $3(6 \%)$ & \\
\hline 4 & 0 & 0 & \\
\hline Single leaflet detachment & $2(4 \%)$ & $2(4 \%)$ & 1.00 \\
\hline Device embolization & 0 & 0 & \\
\hline Urgent heart surgery & 0 & 0 & \\
\hline Major bleeding & $2(4 \%)$ & $1(2 \%)$ & 1.00 \\
\hline Ventilation $>48 \mathrm{~h}$ & $1(2 \%)$ & 0 & 1.00 \\
\hline In-hospital stroke & 0 & $1(2 \%)$ & 1.00 \\
\hline In-hospital death & 0 & 0 & \\
\hline
\end{tabular}

Note: Continuous variables are reported as median [IQR]. Categorical variables are expressed as $n(\%)$.

Abbreviations: IQR, interquartile range; $M R$, mitral regurgitation; $S D$, standard deviation.

\section{4 | DISCUSSION}

This is the first study to investigate methods of reducing radiation exposure during transcatheter edge-to-edge mitral valve repair. As the main finding, we demonstrated that radiation dose during MitraClip implantation can be decreased substantially and safely by feasible adjustments of fluoroscopy modalities. Furthermore, to the best of our knowledge, this study constitutes the largest systematic investigation of radiation exposure during transcatheter mitral valve repair so far.

\section{1 | Radiation exposure during MitraClip implantation}

Although interventional edge-to-edge repair is increasingly used for the treatment of mitral regurgitation, data on the radiation dose applied during the procedure are scarce. One study described a median dose of $8910 \mathrm{cGy} \cdot \mathrm{cm}^{2}$ in a small cohort of seven patients, ${ }^{6}$ another investigation with 42 patients reported an even higher average radiation dose of around $14,600 \mathrm{cGy} \cdot \mathrm{cm}^{2} .{ }^{12}$ With a median dose area product of $2265 \mathrm{cGy} \cdot \mathrm{cm}^{2}$ in the normal dose group, radiation exposure in our cohort is almost one order of magnitude lower. The reason for this discrepancy is most likely the increasing experience and practice with the MitraClip system since its introduction, leading to more targeted deployment of fluoroscopy and reduced fluoroscopy time. Likewise, a registry study reported that device time substantially decreased since the emergence of percutaneous mitral valve repair. ${ }^{13}$ Thus, although derived from a singlecenter study, our findings might best represent the actual radiation exposure in contemporary use of MitraClip implantation. In particular, the results might be helpful in establishing dose reference values for percutaneous mitral valve interventions. Furthermore, we demonstrate that radiation exposure during MitraClip implantation is comparable to or lower than the dose applied during percutaneous coronary interventions, which is reported to range from 3000 to $5000 \mathrm{cGy} \cdot \mathrm{cm}^{2} \cdot{ }^{14,15}$ This finding is reassuring for patient and operator
(A)



(B)



(C)

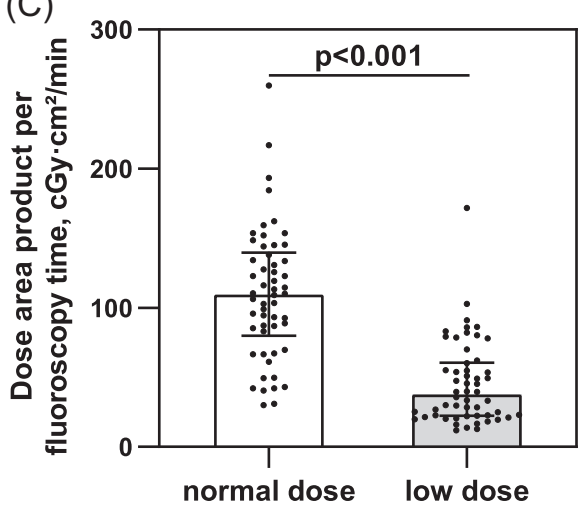

FIGURE 3 Dose area product (A), fluoroscopy time (B), and dose area product per fluoroscopy time (C) during transcatheter mitral valve repair, stratified by fluoroscopy protocol. Bars show median with interquartile range. Dose area product is presented on a logarithmic scale 


\begin{tabular}{lcllll} 
Variable & B & B 95\% Cl & $\boldsymbol{\beta}$ & $\begin{array}{l}\text { Variance } \\
\text { inflation factor }\end{array}$ & $\boldsymbol{p}$ \\
\hline Low dose protocol & -1.03 & {$[-1.20$ to -0.85$]$} & -0.52 & 1.20 & $<0.001$ \\
$\begin{array}{l}\text { Fluoroscopy time (natural } \\
\text { logarithm) }\end{array}$ & 1.13 & {$[0.92-1.34]$} & 0.58 & 1.87 & $<0.001$ \\
\hline Body mass index & 0.06 & {$[0.04-0.08]$} & 0.30 & 1.05 & $<0.001$ \\
\hline Female gender & -0.54 & {$[-0.72$ to -0.36$]$} & -0.26 & 1.21 & $<0.001$ \\
\hline MR Grade 4 at baseline & 0.08 & {$[-0.14$ to 0.31] } & 0.03 & 1.28 & 0.46 \\
\hline No. of implanted devices & -0.11 & {$[-0.31$ to 0.10] } & -0.06 & 1.93 & 0.30 \\
\hline Intercept & 2.92 & & & &
\end{tabular}

Note: Dose area product was entered as its natural logarithm. Results are presented as unstandardized regression coefficient $B$ and standardized regression coefficient $\beta$. Overall model characteristics: $R^{2}=0.84$, adjusted $R^{2}=0.83, F(6,101)=107, p<0.001$, Durbin-Watson statistic $d=1.82$.

Abbreviations: $\mathrm{Cl}$, confidence interval; $\mathrm{MR}$, mitral regurgitation.
TABLE 3 Predictors of dose area product in multiple linear regression
Death, repeat intervention or heart surgery

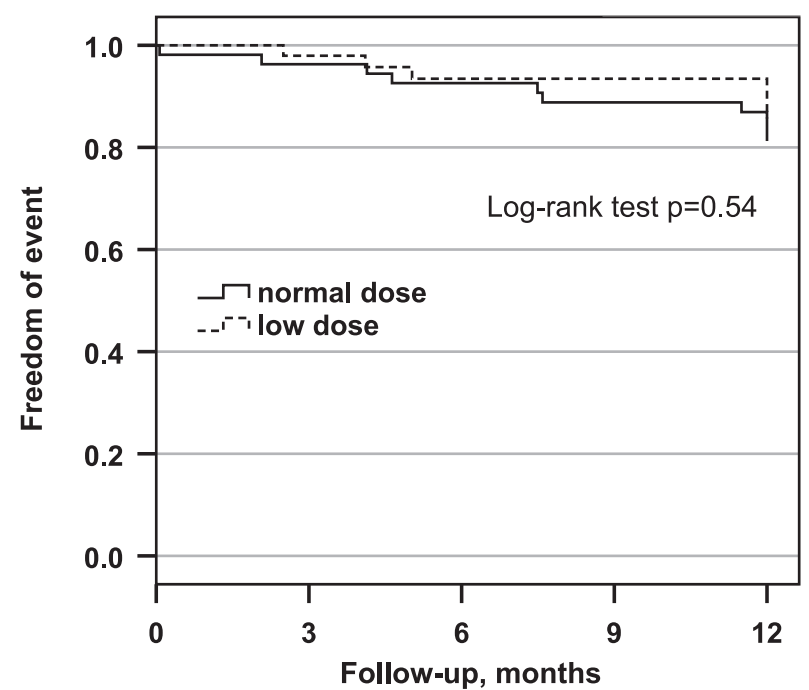

No. at risk

normal dose

low dose

$\begin{array}{ll}54 & 52 \\ 54 & 48\end{array}$

50

47

32

23

FIGURE 4 Freedom of death, repeat intervention or heart surgery during the twelve months follow-up in the normal dose and low dose group

safety, strengthening the safety profile of transcatheter mitral valve repair.

\section{2 | Feasibility of radiation dose reduction}

Our study demonstrated that radiation exposure during MitraClip implantation can be reduced substantially by introducing a dose reduction program. Through optimizing default fluoroscopy settings, we were able to decrease radiation dose applied during the procedure by $64 \%$. The resulting median dose area product of around $700 \mathrm{cGy} \cdot \mathrm{cm}^{2}$ is comparable to the radiation burden of diagnostic coronary angiography. ${ }^{15} \mathrm{~A}$ recent analysis of national registry data including 6064 mitral valve interventions performed in 2020 reported a median dose area product of $1300 \mathrm{cGy} \cdot \mathrm{cm}^{2} \cdot{ }^{16}$ Considering these results, adopting our proposed low dose settings has the potential to reduce radiation exposure in transcatheter mitral valve repair on a large scale. The implemented modifications are simple and do not require a change in $\mathrm{X}$-ray equipment or alterations of the procedure. While all procedures in our study were performed with one specific fluoroscopy setup, it is plausible that comparable dose reduction is also achievable with different X-ray devices from other manufacturers. Similar effects of optimized fluoroscopy settings on radiation exposure were also observed in dose reduction programs for coronary angiography and electrophysiological procedures, ${ }^{11,14}$ strengthening the validity of our findings.

As transcatheter mitral valve repair is mainly guided by transesophageal echocardiography, ${ }^{17}$ the impact of fluoroscopic image quality on the outcome might be limited at first glance. However, several steps in the procedure still necessitate fluoroscopic visualization. Additionally, adjunctive use of fluoroscopy reduces the need for sub-valvular clip manipulation. The latter is considered to be associated with increased procedure times and adverse events. ${ }^{18}$ Therefore, operators may be reluctant to decrease fluoroscopy pulse rate or energy in fear of inferior image quality negatively affecting the procedure. We strongly agree that the goal of reducing radiation exposure must not be pursued at the cost of procedure efficacy and safety. Also, the capability to decrease X-ray intensity without compromising fluoroscopic guidance may depend on the operator's experience and case complexity. However, blinded assessment showed that loss of image quality by lowering fluoroscopy settings is often negligible. ${ }^{19}$ Likewise, our study did not find any evidence of negative effects of the low dose settings on procedural success, safety, or clinical outcome. Considering the capabilities of 3D transesophageal echocardiography, future studies may investigate whether additional reduction of radiation dose is feasible by further limiting the use of fluoroscopy to certain steps of the procedure, guiding grasping and clip deployment exclusively by echocardiography. 
It is natural that reducing fluoroscopy intensity and frame rate decreases radiation dose. However, the extent of reducing radiation exposure must be traded off with the effectiveness of the imaging modality, including a potential negative impact on the procedure. Beyond the results on dose reduction, our study indicates that performing MitraClip implantation using the described fluoroscopy settings is safe and does not prolong the procedure. Thus, our findings might help and encourage other care centers to implement similar dose reduction programs.

\section{3 | Clinical relevance}

lonizing radiation generated by $\mathrm{X}$-ray fluoroscopy has a well-known detrimental effect on human tissue. Acute effects on exposed skin are often self-limiting, but can be severe after long, intense, or repeated fluoroscopy. ${ }^{20}$ More concerning is the inevitable stochastic risk for the induction of malignant disease, which grows linearly with the cumulative applied dose. Importantly, current models do not suggest a threshold dose below which the stochastic risk is absent. ${ }^{21}$ As a consequence, there is consensus to reduce radiation exposure during medical and particularly cardiovascular imaging whenever possible. ${ }^{1,3}$

As a radiation-induced malignancy needs time to develop and become manifest in a person, the excess risk caused by radiation substantially decreases with higher age at the time of exposition. ${ }^{21}$ In this context, one might argue that limiting radiation exposure is of low relevance in the elderly patient population treated with transcatheter mitral valve repair. We consider this conclusion to be wrong for several reasons. First, radiation safety universally mandates medical radiation exposure to be "as low as reasonably achievable" (ALARA principle). ${ }^{3}$ Therefore, if radiation dose during transcatheter mitral valve repair can be decreased without compromising procedural outcome, patient age does not constitute a constraint for reducing radiation exposure. Second, given the trajectory of progress in minimal-invasive techniques, it is reasonable to assume that the future patient population will expand towards a low-risk and younger collective. Furthermore, our findings might also help to achieve comparable radiation reduction in other fluoroscopy-guided percutaneous treatments performed in predominantly mid-aged patients, such as closure of patent foramen ovale. ${ }^{22}$

Third, radiation exposure does not only affect patients, but also medical personnel involved in transcatheter mitral valve repair. Interventional cardiologists are among the highest exposed healthcare workers, with a lifetime attributable risk of cancer of up to 1 in 200 subjects. $^{23}$ In particular, procedures such as MitraClip implantation also require a transesophageal echo operator who receives comparable or higher radiation doses than the catheter operators due to his proximity to the X-ray source. ${ }^{24,25}$ Although protective measures and equipment play a key role in occupational radiation safety, personnel dose levels naturally correlate with patient dose levels. ${ }^{26,27}$ Therefore, while its effect on radiation dose of performing personnel remains to be elucidated in future studies, our proposed low dose protocol might have the potential to reduce occupational radiation exposure in light of the increasing usage of fluoroscopy-guided percutaneous therapies.

\section{4 | Limitations}

Our study might have certain limitations.

(1) As the study was based on an institutional effort to reduce radiation exposure during transcatheter mitral valve repair, the operators were not blinded to which fluoroscopy protocol was being used. Therefore, we cannot rule out that the operator's individual intention to decrease radiation exposure resulted in more targeted deployment of fluoroscopy. Indeed, fluoroscopy time was lower in the low dose group, naturally contributing to the observed reduction of radiation exposure. However, dose area product per fluoroscopy time also substantially decreased after introduction of the low dose protocol, demonstrating the effectiveness of the low dose settings in reducing radiation exposure independent of fluoroscopy time. Furthermore, the low dose protocol remained a strong negative predictor of radiation dose after correcting for fluoroscopy time in multiple linear regression.

(2) Due to its observational nature, patients were not randomly allocated to the low dose and normal dose group. Although both groups showed similar mitral regurgitation severity, cardiac function, and functional status, we cannot rule out that differences in baseline characteristics influenced the results. As the low dose and normal dose group were separated by time, increasing experience with the MitraClip procedure at our center during the inclusion period might have contributed to the observed reduction in radiation exposure. However, transcatheter mitral valve repair was well established at our institution, with every primary operator having performed over 50 procedures at the beginning of study enrollment. Furthermore, DurbinWatson statistics in linear regression remained without evidence of temporal autocorrelation of radiation exposure.

(3) The overall incidence of the combined endpoint during the twelve-months follow up was low, limiting the statistical power to detect differences in midterm prognosis between both groups.

(4) Our study represents results of a single academic tertiary care center. Therefore, its findings might not be fully applicable to other care centers with differing patient population, equipment, or experience.

\section{5 | CONCLUSION}

By implementing a low dose fluoroscopy protocol, radiation exposure during transcatheter edge-to-edge mitral valve repair can be reduced by $64 \%$. Performing transcatheter mitral valve repair with low dose 
fluoroscopy settings is not associated with adverse effects on procedural success, duration, or safety.

\section{ACKNOWLEDGMENT}

Open Access funding enabled and organized by Projekt DEAL.

\section{CONFLICT OF INTERESTS}

K. Debl, C. Meindl, and B. Unsöld received travel support from Abbott Vascular. M.G. Paulus, M. Hamerle, C. Schach, L.S. Maier, and C. Birner declare that there are no conflict of interests.

\section{DATA AVAILABILITY STATEMENT}

The data that support the findings of this study are available from the corresponding author upon reasonable request.

\section{ORCID}

Michael G. Paulus (D) http://orcid.org/0000-0001-9413-885X Christine Meindl D http://orcid.org/0000-0001-7086-0469

\section{REFERENCES}

1. Picano $E$, Vañó $E$, Rehani $M M$, et al. The appropriate and justified use of medical radiation in cardiovascular imaging: a position document of the ESC Associations of Cardiovascular Imaging, Percutaneous Cardiovascular Interventions and Electrophysiology. Eur Heart J. 2014;35:665-672.

2. Bedetti G, Botto N, Andreassi MG, Traino C, Vano E, Picano E. Cumulative patient effective dose in cardiology. Br J Radiol. 2008;81: 699-705.

3. Hirshfeld JW, Ferrari VA, Bengel FM, et al. 2018 ACC/HRS/ $\mathrm{NASCl} / \mathrm{SCAl} / \mathrm{SCCT}$ expert consensus document on optimal use of ionizing radiation in cardiovascular imaging: best practices for safety and effectiveness: a report of the American College of Cardiology Task Force on expert consensus decision pathways. J Am Coll Cardiol, 2018(71):e283-e351.

4. Glower DD, Kar S, Trento A, et al. Percutaneous mitral valve repair for mitral regurgitation in high-risk patients: results of the EVEREST II study. J Am Coll Cardiol. 2014;64:172-181.

5. Stone GW, Lindenfeld J, Abraham WT, et al. Transcatheter mitral-valve repair in patients with heart failure. $N$ Engl J Med. 2018;379:2307-18.

6. Boland JE, Wang LW, Love BJ, Wynne DG, Muller DWM. Radiation dose during percutaneous treatment of structural heart disease. Heart Lung Circ. 2014;23:1075-1083.

7. Feldman T, Kar S, Rinaldi M, et al. Percutaneous mitral repair with the MitraClip system: safety and midterm durability in the initia EVEREST (Endovascular Valve Edge-to-Edge REpair Study) cohort. J Am Coll Cardiol. 2009;54:686-694.

8. Zoghbi WA, Adams D, Bonow RO, et al. Recommendations for noninvasive evaluation of native valvular regurgitation: a report from the American Society of Echocardiography developed in collaboration with the Society for Cardiovascular Magnetic Resonance. J Am Soc Echocardiogr. 2017;30:303-371.

9. Zoghbi WA, Asch FM, Bruce C, et al. Guidelines for the evaluation of valvular regurgitation after percutaneous valve repair or replacement: a report from the American Society of Echocardiography developed in collaboration with the
Society for Cardiovascular Angiography and Interventions, Japanese Society of Echocardiography, and Society for Cardiovascular Magnetic Resonance. J Am Soc Echocardiogr. 2019;32: 431-475.

10. Ebrahimi R, Uberoi A, Treadwell M, Sadrzadeh, Rafie AH. Effect of low-frame invasive coronary angiography on radiation and image quality. Am J Cardiol. 2016;118:195-197.

11. Reissmann B, Maurer T, Wohlmuth $P$, et al. Significant reduction of radiation exposure in cryoballoon-based pulmonary vein isolation. Europace. 2018;20:608-613.

12. Sündermann $\mathrm{SH}$, Biaggi $\mathrm{P}$, Grünenfelder J, et al. Safety and feasibility of novel technology fusing echocardiography and fluoroscopy images during MitraClip interventions. Eurolntervention. 2014;9: 1210-1216.

13. Rahhab Z, Kortlandt FA, Velu JF, et al. Current MitraClip experience, safety and feasibility in the Netherlands. Neth Heart $J$. 2017;25:394-400.

14. Abdelaal E, Plourde G, MacHaalany J, et al. Effectiveness of low rate fluoroscopy at reducing operator and patient radiation dose during transradial coronary angiography and interventions. JACC CardiovasC Interv. 2014;7:567-574.

15. Seiffert M, Ojeda F, Müllerleile K, et al. Reducing radiation exposure during invasive coronary angiography and percutaneous coronary interventions implementing a simple four-step protocol. Clin Res Cardiol. 2015;104:500-506.

16. German Institute for Quality Assurance and Transparency in Health Care. Kathetergestützte isolierte Mitralklappeneingriffe [Catheter-based isolated mitral valve interventions]. 2021.

17. Silvestry FE, Rodriguez LL, Herrmann HC, et al. Echocardiographic guidance and assessment of percutaneous repair for mitral regurgitation with the Evalve MitraClip: lessons learned from EVEREST I. J Am Soc Echocardiogr. 2007;20: 1131-1140.

18. Singh GD, Rogers JH, Chen S, et al. Adjunctive use of fluoroscopy during MitraClip implantation reduces procedural complexity: The parallax technique. Catheter Cardiovasc Interv. 2020; 97:745-754.

19. Pyne CT, Gadey G, Jeon C, Piemonte T, Waxman S, Resnic F. Effect of reduction of the pulse rates of fluoroscopy and CINEacquisition on $\mathrm{x}$-ray dose and angiographic image quality during invasive cardiovascular procedures. Circ Cardiovasc Interv. 2014; 7:441-446.

20. Balter S, Hopewell JW, Miller DL, Wagner LK, Zelefsky MJ. Fluoroscopically guided interventional procedures: a review of radiation effects on patients' skin and hair. Radiology. 2010;254: 326-341.

21. National Research Council (U.S.). Health risks from exposure to low levels of ionizing radiation: BEIR VII, Phase 2. 7, phase 2. Washington, D.C: National Academies Press. 2006.

22. Alushi B, Lauten A, Cassese $S$, et al. Patent foramen ovale closure versus medical therapy for prevention of recurrent cryptogenic embolism: updated meta-analysis of randomized clinical trials. Clin Res Cardiol. 2018;107:788-98.

23. Venneri L, Rossi F, Botto N, et al. Cancer risk from professional exposure in staff working in cardiac catheterization laboratory: insights from the National Research Council's Biological Effects of lonizing Radiation VII Report. Am Heart J. 2009;157: 118-124.

24. Crowhurst JA, Scalia GM, Whitby M, et al. Radiation exposure of operators performing transesophageal echocardiography during 
percutaneous structural cardiac interventions. J Am Coll Cardiol. 2018;71:1246-54.

25. Salaun E, Carles S, Bigand E, et al. High radiation exposure of the imaging specialist during structural heart interventions with echocardiographic guidance. JACC Cardiovasc Interv. 2017;10: 626-627.

26. Tsapaki V, Kottou S, Vano E, et al. Correlation of patient and staff doses in interventional cardiology. Radiat Prot Dosimetry. 2005;117: 26-29.

27. Antic V, Ciraj-Bjelac O, Rehani M, Aleksandric S, Arandjic D, Ostojic M. Eye lens dosimetry in interventional cardiology: results of staff dose measurements and link to patient dose levels. Radiat Prot Dosimetry. 2013;154:276-284.

\section{SUPPORTING INFORMATION}

Additional supporting information may be found in the online version of the article at the publisher's website.

How to cite this article: Paulus MG, Meindl C, Hamerle M, et al. Reduction of radiation exposure during transcatheter edge-to-edge mitral valve repair. Catheter Cardiovasc Interv. 2021;1-9. doi:10.1002/ccd.30046 